\title{
ON THE STRONG LAW OF LARGE NUMBERS AND THE CENTRAL LIMIT THEOREM FOR MARTINGALES $\left.{ }^{(}\right)$
}

\author{
BY \\ MIKLÓS CSÖRGÖ
}

1. Introduction. Let $X_{1}, X_{2}, \ldots$ be random variables on some probability space $(\Omega, \mathscr{B}, P)$ satisfying

$$
E\left(X_{1}\right)=0, \quad E\left(X_{n} \mid X_{1}, \ldots, X_{n-1}\right)=0, \quad n \geqq 2,
$$

with probability 1 . Define

$$
S_{n}=X_{1}+\cdots+X_{n}
$$

A sequence of random variables $\left\{X_{k}\right\}$ satisfying (1.1) will be called absolutely fair (a term introduced by Feller in VI.12 of his book [5]).

The partial sums $\left\{S_{n}\right\}$ of an absolutely fair sequence of random variables form a martingale, that is

$$
E\left(S_{n} \mid S_{1}, \ldots, S_{n-1}\right)=S_{n-1}, \quad n \geqq 1 \quad\left(S_{0}=0\right),
$$

with probability 1 . In fact, a sequence of partial sums of random variables whose expectations exist is a martingale if and only if (1.1) is true (see e.g. VI.12 of Feller's book [5], or 11.7 of Doob's book [4]), and then we have $E\left(X_{n}\right)=0$ for all $n$.

Also, if $E\left(S_{n}^{2}\right)<\infty$ for all $n$, the sequence of squares of partial sums of an absolutely fair sequence of random variables is a submartingale, that is

$$
E\left(S_{n}^{2} \mid S_{1}^{2}, \ldots, S_{n-1}^{2}\right) \geqq S_{n-1}^{2},
$$

with probability 1 , for $f(x)=x^{2}$ is a continuous convex function of the real variable $x$.

The following theorem is well known (see e.g. VII.8 of [5]).

THEOREM 1. Let $\left\{X_{k}\right\}$ satisfy (1.1) and define $\left\{S_{n}\right\}$ by (1.2). If $b_{1}<b_{2}<\cdots \rightarrow \infty$ and

$$
\sum_{1}^{\infty} b_{k}^{-2} E\left(X_{k}^{2}\right)<\infty
$$

then

$$
\lim _{n \rightarrow \infty} b_{n}^{-1} S_{n}=0, \quad \text { with probability } 1
$$

As a corollary we get the law of large numbers for martingales: if $\sum_{1}^{\infty} n^{-2} E\left(X_{n}^{2}\right)$

Received by the editors January 13, 1967.

(1) Work supported in part by The Canadian Mathematical Congress Summer Research Institute at Queen's University, summer 1966 and 1967. 
$<\infty$ then $n^{-1} S_{n} \rightarrow 0$ with probability 1 , a statement which was first proved by Kolmogorov for sums of mutually independent random variables.

Given the condition (1.5) we also have that

$$
\sum_{1}^{\infty} b_{k}^{-1} X_{k} \text { converges with probability } 1 \text {, }
$$

by the martingale convergence theorem (see e.g. Theorem 1, p. 236 of [5]). Further, (1.7) and Kronecker's lemma imply Theorem 1 of this section, a fact which we are not going to exploit here.

One of the aims of this paper is to prove Theorem 1 and some generalizations of it using only a generalized form of Kolmogorov's inequality for martingales (see e.g. [5, p. 235] for Kolmogorov's inequality for martingales). This is done in $\S 2$ (Inequalities 1, 2 and 3; Theorems 2 and 3).

Let $\left\{\nu_{n}\right\}$ be a sequence of positive integer valued random variables on $(\Omega, \mathscr{B}, P)$. Define the randomized sum

$$
S_{v_{n}}=X_{1}+\cdots+X_{v_{n}} .
$$

In $\$ 2$ again, we are going to prove some strong laws of large numbers for randomized partial sums $\left\{S_{v_{n}}\right\}$ of absolutely fair random variables (Theorem 4).

Let again $\left\{X_{k}\right\}$ be an absolutely fair sequence of random variables satisfying also

$$
E\left(X_{1}^{2}\right)=\sigma_{1}^{2}, \quad E\left(X_{k}^{2} \mid X_{1}, \ldots, X_{k-1}\right)=\sigma_{k}^{2}, \quad k \geqq 2,
$$

with probability 1 , where $\sigma_{1}, \sigma_{2}, \ldots$ are nonnegative constants. Define

$$
\hat{\sigma}_{n}^{2}=\sigma_{1}^{2}+\cdots+\sigma_{n}^{2} .
$$

Let $\mathscr{B}_{j}$ be the Borel field of $\omega$ sets, $\omega \in \Omega$, determined by conditions on $X_{1}, \ldots, X_{j}$. Then (1.1) and (1.9) imply

$$
E\left(S_{j}^{2}-\hat{\sigma}_{j}^{2} \mid \mathscr{B}_{j}\right)=S_{j-1}^{2}-\hat{\sigma}_{j-1}^{2}, \quad j>1,
$$

with probability 1. In other words, if (1.1) and (1.9) are true, the process $\left\{S_{j}^{2}-\hat{\sigma}_{j}^{2}, \mathscr{B}_{j}, j \leqq n\right\}$ is a martingale. Conversely, if $(1.1)$ is true and if $\left\{S_{j}^{2}-\hat{\sigma}_{j}^{2}, \mathscr{B}_{j}, j \leqq n\right\}$ is a martingale then (1.9) is true. In particular, (1.9) is true if the $X_{k}$ 's are mutually independent, with zero expectations and finite variances. Condition (1.9) also implies that $E\left(X_{k}^{2}\right)=\sigma_{k}^{2}, k \geqq 1$, and $E\left(\prod_{1}^{k} X_{j}^{2}\right)=\prod_{1}^{k} E\left(X_{j}^{2}\right)$.

Doob [4, pp. 382-384] shows that the central limit theorem is applicable to sums of random variables $\left\{X_{k}\right\}$ satisfying (1.1) and (1.9) much as it is to sums of mutually independent random variables and remarks that this fact was first observed by P. Lévy. We are going to show that the central limit theorem is applicable to randomly selected sums of an absolutely fair sequence of random variables too, provided (1.9) is true. This statement is going to be made precise and proved in $\S 3$ (Theorems 6 and 8). Also in $\$ 3$ we are going to show that, when properly normed, partial sums of an absolutely fair sequence $\left\{X_{k}\right\}$ satisfying (1.9) form a 
mixing sequence with their limiting unit normal distribution (this terminology and statement is going to be made precise there; Theorems 9 and 13), a fact which implies some further results about randomly selected partial sums of these random variables (Theorems 10,12, 14 and 15). Theorems 16 and 17 of $\S 3$ are also consequences of this mixing property of partial sums of absolutely fair random variables.

2. Some inequalities for submartingales and martingales and some strong laws of large numbers for martingales.

INEQUALITY 1 (a generalization of Kolmogorov's inequality for submartingales). Let $\left\{Y_{k}\right\}$ be a submartingale sequence of random variables and $Y_{k} \geqq 0$ for all $k$. If $\left\{c_{k}\right\}$ is a nonincreasing sequence of positive constants, then for any positive integers $m$ and $n$ with $m<n$ and arbitrary $x>0$,

$$
P\left\{\max _{m \leqq k \leqq n} c_{k}^{2} Y_{k} \geqq x\right\} \leqq \frac{1}{x}\left\{\sum_{k=m}^{n-1}\left(c_{k}^{2}-c_{k+1}^{2}\right) E\left(Y_{k}\right)+c_{n}^{2} E\left(Y_{n}\right)\right\} .
$$

If in (2.1) all $c_{k}=1$ and $m=1$, we get Kolmogorov's inequality for submartingales $[5,(8.6)$, p. 235].

Proof of Inequality 1. For fixed $x>0$ let $A_{r}, r=m, m+1, \ldots, n$, be the $\omega$ set, $\omega \in \Omega$, for which $c_{r}^{2} Y_{r}(\omega)$ is the first $c_{j}^{2} Y_{j}(\omega)$ with $c_{j}^{2} Y_{j}(\omega) \geqq x$. That is, we have

$$
\begin{aligned}
& A_{r}=\left\{\omega: c_{r}^{2} Y_{r}(\omega) \geqq x\right\}, \quad r=m, \\
& A_{r}=\left\{\omega: c_{j}^{2} Y_{j}(\omega)<x, m \leqq j<r ; c_{r}^{2} Y_{r}(\omega) \geqq x\right\}, \quad m<r \leqq n .
\end{aligned}
$$

Obviously, the $A_{r}$ 's are disjoint $\omega$ sets (mutually exclusive events) and we have

$$
P\left\{\max _{m \leqq k \leqq n} c_{k}^{2} Y_{k} \geqq x\right\}=\sum_{k=m}^{n} P\left(A_{k}\right) \text {. }
$$

Define

$$
J=\sum_{k=m}^{n-1}\left(c_{k}^{2}-c_{k+1}^{2}\right) Y_{k}+c_{n}^{2} Y_{n}
$$

Thus, in order to prove (2.1), we have to show

$$
\sum_{k=m}^{n} P\left(A_{k}\right) \leqq \frac{1}{x} E(J) \text {. }
$$

Now, because we assume $Y_{k} \geqq 0$ for all $k$, we have

$$
E(J) \geqq \sum_{r=m}^{n} E\left(J \mid A_{r}\right) P\left(A_{r}\right) .
$$

Also, for $m \leqq r \leqq n$,

$$
E\left(J \mid A_{r}\right) \geqq \sum_{k=r}^{n-1}\left(c_{k}^{2}-c_{k+1}^{2}\right) E\left(Y_{k} \mid A_{r}\right)+c_{n}^{2} E\left(Y_{n} \mid A_{r}\right) .
$$

So far this proof differs only notationally from Rényi's proof of the HájekRényi inequality in [6] to the extent that in his case $Y_{k}$ of (2.3) is equal to the square of the sum of $k$ mutually independent random variables. 
Now we verify

$$
E\left(Y_{k} \mid A_{r}\right) \geqq E\left(Y_{r} \mid A_{r}\right) \geqq x / c_{r}^{2}, \quad m \leqq r \leqq k \leqq n .
$$

The last inequality of (2.7) follows from the definition of $A_{r}$ and, if $\chi_{r}$ is the indicator function of the $\omega$ set $A_{r}$,

$$
\begin{aligned}
E\left(Y_{k} \mid A_{r}\right) & =\frac{1}{P\left(A_{r}\right)} E\left(\chi_{r} Y_{k}\right)=\frac{1}{P\left(A_{r}\right)} E\left(E\left(\chi_{r} Y_{k} \mid Y_{1}, \ldots, Y_{r}\right)\right) \\
& =\frac{1}{P\left(A_{r}\right)} E\left(\chi_{r} E\left(Y_{k} \mid Y_{1}, \ldots, Y_{r}\right)\right) \geqq \frac{1}{P\left(A_{r}\right)} E\left(\chi_{r} Y_{r}\right)=E\left(Y_{r} \mid A_{r}\right),
\end{aligned}
$$

on using the submartingale property of the sequence $\left\{Y_{k}\right\}$. This, through (2.6) and (2.5), implies (2.4) and Inequality 1 is proved.

INEQUALITY 2 (a generalization of Kolmogorov's inequality for martingales). Let $\left\{X_{k}\right\}$ be a sequence of random variables satisfying (1.1) and define $\left\{S_{n}\right\}$ by (1.2). If $E\left(S_{n}^{2}\right)<\infty$ for all $n$, and $\left\{c_{n}\right\}$ is a nonincreasing sequence of positive constants, then for any positive integers $m$ and $n$ with $m<n$ and arbitrary $\varepsilon>0$,

$$
\begin{aligned}
P\left\{\max _{m \leqq k \leqq n} c_{k}\left|S_{k}\right| \geqq \varepsilon\right\} & \leqq \frac{1}{\varepsilon^{2}}\left\{\sum_{k=m}^{n-1}\left(c_{k}^{2}-c_{k+1}^{2}\right) E\left(S_{k}^{2}\right)+c_{n}^{2} E\left(S_{n}^{2}\right)\right\} \\
& =\frac{1}{\varepsilon^{2}}\left\{c_{m}^{2} \sum_{k=1}^{m} E\left(X_{k}^{2}\right)+\sum_{k=m+1}^{n} c_{k}^{2} E\left(X_{k}^{2}\right)\right\} .
\end{aligned}
$$

In case the $X_{k}$ 's are mutually independent (2.8) is known as the Hájek-Rényi inequality (see e.g. [6]), and then the equality of the two expressions on the righthand side of (2.8) is obvious. If in (2.8) all $c_{k}=1$ and $m=1$, we get Kolmogorov's inequality for martingales ([5, p. 235]; also [4, p. 315]), and if the $X_{k}$ 's are mutually independent, the original Kolmogorov inequality.

Proof of Inequality 2. $\left\{S_{n}\right\}$ is a martingale sequence and $\left\{S_{n}^{2}\right\}$ is a submartingale sequence for we assume $E\left(S_{n}^{2}\right)<\infty$. Let $Y_{k}=S_{k}^{2}$ and $x=\varepsilon^{2}$ in (2.1) and the first line of (2.8) follows. The second form of the right-hand side of $(2.8)$ is implied by the absolute fairness of the sequence $\left\{X_{n}\right\}$. To see this, we consider

$$
E\left(S_{k}^{2}\right)=E\left(E\left(S_{k}^{2} \mid X_{1}, \ldots, X_{k-1}\right)\right), \quad k=1, \ldots, n \quad\left(X_{0}=0\right) .
$$

Now

$$
\begin{aligned}
E\left(S_{k}^{2} \mid X_{1}, \ldots, X_{k-1}\right) & =E\left\{\left(S_{k-1}+X_{k}\right)^{2} \mid X_{1}, \ldots, X_{k-1}\right\} \\
& =S_{k-1}^{2}+E\left(X_{k}^{2} \mid X_{1}, \ldots, X_{k-1}\right)
\end{aligned}
$$

by (1.1), $k=1, \ldots, n\left(S_{0}=0\right)$, and then (2.9) implies

$$
E\left(S_{k}^{2}\right)=E\left(S_{k-1}^{2}\right)+E\left(X_{k}^{2}\right),
$$

and the second form of the right-hand side of (2.8) follows.

As a byproduct of $(2.10)$ we note that it shows that the sequence $\left\{S_{k}^{2}\right\}$ is a submartingale. Also, if we assume that the absolutely fair sequence $\left\{X_{k}\right\}$ satisfies the 
hypothesis (1.9) too and if $\hat{\sigma}_{n}^{2}$ is as in (1.10), then (2.10) shows that the sequence $\left\{S_{k}^{2}-\hat{\sigma}_{k}^{2}\right\}$ is a martingale, that is

$$
E\left(S_{k}^{2}-\hat{\sigma}_{k}^{2} \mid X_{1}, \ldots, X_{k-1}\right)=S_{k-1}^{2}-\hat{\sigma}_{k-1}^{2}, \quad k>1,
$$

with probability 1 .

Inequality 2 can be generalized to an arbitrary sequence of random variables as follows.

INEQUALITY 3. Let $\left\{\xi_{k}\right\}$ be a sequence of random variables with $E\left(\left|\xi_{k}\right|^{r}\right)<\infty$, $r$ a fixed positive integer. Define

$$
V_{n}=\sum_{1}^{n}\left[\xi_{k}^{r}-E\left(\xi_{k}^{r} \mid \xi_{1}, \ldots, \xi_{k-1}\right)\right], \quad \xi_{0}=0
$$

and assume $E\left(V_{n}^{2}\right)<\infty$. If the sequence $\left\{c_{k}\right\}, m, n$ and $\varepsilon$ are as in Inequality 2 , then

$$
\begin{aligned}
P\left\{\max _{m \leqq k \leqq n} c_{k}\left|V_{k}\right| \geqq \varepsilon\right\} \leqq & \frac{1}{\varepsilon^{2}}\left\{\sum_{k=m}^{n-1}\left(c_{k}^{2}-c_{k+1}^{2}\right) E\left(V_{k}^{2}\right)+c_{n}^{2} E\left(V_{n}^{2}\right)\right\} \\
= & \frac{1}{\varepsilon^{2}}\left[c_{m}^{2} \sum_{k=1}^{m} E\left\{\left[\xi_{k}^{r}-E\left(\xi_{k}^{r} \mid \xi_{1}, \ldots, \xi_{k-1}\right)\right]^{2}\right\}\right. \\
& \left.\quad+\sum_{k=m+1}^{n} c_{k}^{2} E\left\{\left[\xi_{k}^{r}-E\left(\xi_{k}^{r} \mid \xi_{1}, \ldots, \xi_{k-1}\right)\right]^{2}\right\}\right] .
\end{aligned}
$$

Proof of Inequality 3. The summands of $V_{n}$ have the property (1.1), that is they are absolutely fair random variables. Replace $\left\{X_{k}\right\}$ by $\left\{\xi_{k}^{r}-E\left(\xi_{k}^{r} \mid \xi_{1}, \ldots, \xi_{k-1}\right)\right\}$ and $\left\{S_{n}\right\}$ by $\left\{V_{n}\right\}$ in Inequality 2 . The second form of (2.14) follows from an argument concerning $E\left(V_{k}^{2}\right)$ similar to that concerning $E\left(S_{k}^{2}\right)$ in (2.9), (2.10) and (2.11) above.

Letting $n \rightarrow \infty$ and assuming $\sum_{1}^{\infty} c_{k}^{2} E\left(X_{k}^{2}\right)<\infty$ in (2.8), we get

$$
\lim _{m \rightarrow \infty} P\left\{\sup _{m \leqq k} c_{k}\left|S_{k}\right| \geqq \varepsilon\right\}=0
$$

and, equivalently,

$$
\lim _{n \rightarrow \infty} c_{n} S_{n}=0, \quad \text { with probability } 1 .
$$

This, with $c_{k}=b_{k}^{-1}$, is the statement of Theorem 1 of $\S 1$.

It is perhaps of some interest to note here that if $\left\{X_{k}\right\}$ is a sequence of mutually orthogonal random variables with $E\left(X_{k}\right)=0$ and $E\left(X_{k}^{2}\right)$ finite for all $k$, then $n^{-1} S_{n}$ $\rightarrow 0$ with probability 1 if $\sum_{1}^{\infty} n^{-2} \log ^{2} n E\left(X_{n}^{2}\right)<\infty$ [4, p. 158], a condition which can be replaced by $\sum_{1}^{\infty} n^{-2} E\left(X_{n}^{2}\right)<\infty$ if we also assume that the sequence $\left\{X_{k}\right\}$ of mutually orthogonal random variables is absolutely fair. In fact, we do not need then the orthogonality assumption any more. Thus, as far as the strong law of large numbers is concerned, absolutely fair random variables behave like mutually independent ones, while mutually orthogonal random variables require more severe restrictions on their variances in order to have the strong law of large numbers hold. 
Theorem 1 can be generalized to an arbitrary sequence of random variables as follows.

THEOREM 2. Let $\left\{\xi_{k}\right\}$ be a sequence of random variables and assume, for a fixed positive integer $r$, that $E\left(\left|\xi_{k}\right|^{r}\right)<\infty$. Define $V_{n}$ as in (2.13). If $E\left(V_{n}^{2}\right)<\infty, b_{1}<b_{2}$ $<\cdots \rightarrow \infty$, and

$$
\sum_{1}^{\infty} b_{k}^{-2} E\left\{\left[\xi_{k}^{r}-E\left(\xi_{k}^{r} \mid \xi_{1}, \ldots, \xi_{k-1}\right)\right]^{2}\right\}<\infty
$$

then

$$
\lim _{n \rightarrow \infty} b_{n}^{-1} V_{n}=0 \text {, with probability } 1 \text {. }
$$

Theorem 2 can be proved from Inequality 3 exactly the same way as Theorem 1 above from Inequality 2 .

THEOREM 3. Retain the assumptions of Theorem 2 about the sequences $\left\{\xi_{n}\right\}$ and $\left\{V_{n}\right\}$, and assume, for a fixed positive integer $r$, that $E\left\{\xi_{k}^{r} \mid \xi_{1}, \ldots, \xi_{k-1}\right\}=\mu_{k}^{r}$, constants for $k=1,2, \ldots$ Let $\mu_{k}^{r} \geqq 0$ if $r$ is an odd positive integer, and let $\sum_{j=1}^{n} \mu_{j}^{r} \rightarrow \infty$ as $n \rightarrow \infty$ and $r$ even or odd. If

$$
\sum_{k=1}^{\infty} \frac{E\left(\left(\xi_{k}^{r}-\mu_{k}^{r}\right)^{2}\right)}{\left(\sum_{j=1}^{k} \mu_{j}^{r}\right)^{2}}<\infty
$$

then

$$
\lim _{n \rightarrow \infty} \frac{\sum_{1}^{n} \xi_{k}^{r}}{\sum_{1}^{n} \mu_{k}^{r}}=1, \quad \text { with probability } 1
$$

Theorem 3, with $c_{k}=\left(\sum_{1}^{k} \mu_{j}^{r}\right)^{-1}$, follows from Inequality 3 exactly the same way as Theorem 1 from Inequality 2 .

If $r=1$ and the sequence $\left\{\xi_{k}\right\}$ is absolutely fair to start with, then Theorem 2 reduces to Theorem 1 . Theorems 2 and 3 are well known for partial sums of mutually independent random variables. The assumption $E\left\{\xi_{k}^{r} \mid \xi_{1}, \ldots, \xi_{k-1}\right\}=\mu_{k}^{r}$, $k=1,2, \ldots\left(\xi_{0}=0\right)$, of Theorem 3 implies $E\left\{\xi_{k}^{r}\right\}=\mu_{k}^{r}, k=1,2, \ldots$ and also that $E\left(\prod_{j=1}^{k} \xi_{j}^{r}\right)=\prod_{j=1}^{k} E\left(\xi_{j}^{r}\right)$. This is certainly true in case of independent random variables but it does not, of course, imply independence.

Conditions (2.17) and (2.19) imply the convergence of

$$
\sum_{1}^{\infty} b_{k}^{-1}\left[\xi_{k}^{r}-E\left(\xi_{k}^{r} \mid \xi_{1}, \ldots, \xi_{k-1}\right)\right] \text { and } \sum_{1}^{\infty}\left(\sum_{1}^{k} \mu_{j}^{r}\right)^{-1}\left(\xi_{k}^{r}-\mu_{k}^{r}\right)
$$

respectively, with probability 1 .

This follows immediately from the martingale convergence theorem, for the partial sums of the infinite series of (2.21) form a martingale.

Let $\left\{\nu_{n}\right\}$ be a sequence of positive integer valued random variables on $(\Omega, \mathscr{B}, P)$, and let $v_{n} \rightarrow \infty$ with probability 1 as $n \rightarrow \infty$. Define $S_{v_{n}}$ as in (1.8). Similarly, define randomized partial sums for the series occuring in Theorems 2 and 3, (1.7), and 
(2.21). Then, given the conditions of Theorems 1,2 and 3 respectively and $v_{n} \rightarrow \infty$ with probability 1 as $n \rightarrow \infty$, we have

THEOREM 4. With probability 1 the following statements are true:

$$
\begin{aligned}
& \lim _{n \rightarrow \infty} b_{v_{n}}^{-1} S_{v_{n}}=\lim _{n \rightarrow \infty} b_{v_{n}}^{-1} V_{v_{n}}=0, \\
& \lim _{n \rightarrow \infty} \frac{\sum_{1}^{v_{n}} \xi_{k}^{r}}{\sum_{1}^{v_{n}} \mu_{k}^{r}}=1 ;
\end{aligned}
$$

also, the randomized partial sums of (1.7) and (2.21) converge with probability 1 (we have e.g. $\sum_{1}^{v_{n}} b_{k}^{-1} X_{k}$ converges with probability 1 as $n \rightarrow \infty$ ), given the conditions (1.5), (2.17) and (2.19).

Proof of Theorem 4. We are going to show here that, under the conditions of Theorem 1 and assuming $\nu_{n} \rightarrow \infty$ with probability 1 as $n \rightarrow \infty$, we have $\lim _{n \rightarrow \infty} b_{v_{n}}^{-1} S_{v_{n}}=0$ with probability 1 . The statement of Theorem 4 about its other random variables can be proved exactly the same way.

Consider the $\omega$-set

$$
B_{1}=\left\{\omega: b_{v_{n}}^{-1} S_{v_{n}} \rightarrow 0, v_{n} \rightarrow \infty \text { as } n \rightarrow \infty\right\},
$$

and select an element $\omega$ from $B_{1}$. There exists then an $\varepsilon(\omega)>0$ such that for every $n$ we can find an integer $k(n) \geqq n$ and

$$
\left|b_{v_{k(n)}}^{-1} S_{v_{k(n)}}(\omega)\right| \geqq \varepsilon .
$$

As $n \rightarrow \infty$, an ordered set of infinitely many integers $k(n)$ is produced and $k(n) \rightarrow \infty$. Let us put $m_{n}=\nu_{k(n)}(\omega)$, where $\omega \in B_{1}$. Then $m_{n} \rightarrow \infty$ as $n \rightarrow \infty$. By (2.24), we have

$$
\left|b_{m_{n}}^{-1} S_{m_{n}}(\omega)\right| \geqq \varepsilon,
$$

for the sequence $\left\{b_{m_{n}}^{-1} S_{m_{n}}(\omega)\right\}$, and $\omega$ is an element of

$$
B_{2}=\left\{\omega: b_{n}^{-1} S_{n} \nrightarrow 0, \nu_{n} \rightarrow \infty \text { as } n \rightarrow \infty\right\} \text {. }
$$

Thus $B_{1} \subseteq B_{2}$, and $P\left(B_{2}\right)=0$ by Theorem 1 .

The proof of Theorem 4 suggests the following general theorem.

Proposition. Let $\left\{Z_{n}\right\}$ be a sequence of random variables for which the strong law of large numbers holds, that is, $\lim _{n \rightarrow \infty} Z_{n}=0$ with probability 1 . Let $\left\{\nu_{n}\right\}$ be a sequence of positive integer valued random variables and let $\nu_{n} \rightarrow \infty$ with probability 1 as $n \rightarrow \infty$. Then the randomized sequence of random variables $\left\{Z_{v_{n}}\right\}$ also satisfies the strong law of large numbers, that is $\lim _{n \rightarrow \infty} Z_{v_{n}}=0$ with probability 1 .

3. The central limit theorem for the sum of a random number of absolutely fair random variables. Let $X_{1}, X_{2}, \ldots$ be a sequence of random variables on some probability space $(\Omega, \mathscr{B}, P)$ and define $\left\{S_{n}\right\}$ by (1.2). We say the sequence $\left\{X_{k}\right\}$ satisfies the central limit theorem with norming factors $\left\{b_{n}\right\}$ if

$$
\lim _{n \rightarrow \infty} P\left\{S_{n} / b_{n} \leqq x\right\}=\Phi(x),
$$


where $\Phi(x)$ is the unit normal distribution function and the $b_{n}$ 's are positive real numbers.

Let $\left\{\nu_{n}\right\}$ be a sequence of positive integer valued random variables on $(\Omega, \mathscr{B}, P)$. It is frequently of interest to know whether $S_{v_{n}}$ as defined in (1.8) is approximately normal for large $n$. This question is answered by the following special case of a theorem of Anscombe [1] which we now formulate as needed in this paper.

Theorem 5 (ANSCOMBe). Let $\left\{X_{k}\right\}$ satisfy the central limit theorem with norming factors $\left\{b_{n}\right\}$ and assume that for any positive $\varepsilon$ and $\eta$, there exist a positive $c$ and an integer $n_{0}$ such that if $n \geqq n_{0}$ then

$$
P\left\{\max _{|m-n| \leqq n c}\left|S_{n}-S_{m}\right| \geqq \varepsilon b_{n}\right\}<\eta .
$$

Then, if $\nu_{n} / n$ converges in probability to 1 ,

$$
\lim _{n \rightarrow \infty} P\left\{S_{v_{n}} / b_{v_{n}} \leqq x\right\}=\Phi(x) .
$$

First we are going to prove here

THEOREM 6. Let $\left\{X_{k}\right\}$ be an absolutely fair sequence of random variables and assume $\sigma_{k}^{2}=\sigma_{1}^{2}>0, k \geqq 2$, in (1.9). If $\nu_{n} / n$ converges in probability to 1 then

$$
\lim _{n \rightarrow \infty} P\left\{S_{v_{n}} / \nu_{n}^{1 / 2} \sigma_{1} \leqq x\right\}=\Phi(x) .
$$

Proof of Theorem 6. Using the language of (3.1) it follows from Doob's discussion [4, pp. 382-384] that the above sequence $\left\{X_{k}\right\}$ satisfies the central limit theorem with norming factors $\left\{n^{1 / 2} \sigma_{1}\right\}$; that is, we have

$$
\lim _{n \rightarrow \infty} P\left\{S_{n} / n^{1 / 2} \sigma_{1} \leqq x\right\}=\Phi(x) .
$$

Thus, according to Theorem 5 , in order to have (3.4) we will only have to verify that our sequence $\left\{X_{k}\right\}$ of absolutely fair random variables with $E\left(X_{k}^{2} \mid X_{1}, \ldots, X_{k-1}\right)$ $=\sigma_{1}^{2}>0, k \geqq 1\left(X_{0}=0\right)$ satisfies the hypothesis (3.2).

Let $[x]$ denote the integral part of the real number $x$ and consider

$$
\begin{aligned}
& P\left\{\max _{|m-n| \leqq n c} \mid S_{n}-\right.\left.S_{m} \mid \geqq \varepsilon n^{1 / 2} \sigma_{1}\right\} \\
&=P\left\{\max _{[n(1-c)] \leqq m \leqq n n(1+c)]}\left|S_{n}-S_{m}\right| \geqq \varepsilon n^{1 / 2} \sigma_{1}\right\} \\
& \leqq \\
& P\left\{\max _{1 \leqq m \leqq(n(1+c)]}\left|S_{n}-S_{m}\right| \geqq \varepsilon n^{1 / 2} \sigma_{1}\right\} \\
& \leqq \frac{1}{\varepsilon^{2} n \sigma_{1}^{2}} E\left\{\left(S_{n}-S_{[n(1+c))}\right)^{2}\right\}=\frac{1}{\varepsilon^{2} n \sigma_{1}^{2}}\left\{E\left(S_{[n(1+c))}^{2}\right)-E\left(S_{n}^{2}\right)\right\},
\end{aligned}
$$

where the last inequality follows from Kolmogorov's inequality for martingales ((2.8) with all $c_{k}=1$ and $\left.m=1\right)$, and the last equality follows from the martingale property of $\left\{S_{n}\right\}$. Taking into consideration that $E\left(S_{[n(1+c)]}^{2}\right)-E\left(S_{n}^{2}\right)=[c n] \sigma_{1}^{2}$, it 
follows now that (3.2) is satisfied for the sequence $\left\{X_{k}\right\}$ of Theorem 6 , and this completes its proof too.

It is clear that the assumption $\nu_{n} / n$ converges in probability to 1 in Theorem 6 can be replaced by the assumption $\nu_{n} / f(n)$ converges in probability to 1 , where $f(n)$ is an arbitrary real valued function which increases monotonically to $\infty$ as $n \rightarrow \infty$. We could, for example, take $f(n)=\lambda n$, where $\lambda$ is a positive constant, and then we would have $\nu_{n} / n$ converges in probability to $\lambda$ instead of 1 in Theorem 6 .

Now we formulate a theorem of P. Lévy [4, p. 383].

THEOREM 7 (P. LÉVY). Let $\left\{X_{k}\right\}$ be an absolutely fair sequence of random variables satisfying (1.9). Define $\hat{\sigma}_{n}^{2}$ as in (1.10) and assume $\hat{\sigma}_{n}^{2}>0$. If for large $n$ the random variables $\left\{X_{k} / \hat{\sigma}_{n}\right\}$ are infinitesimal in the sense of III.4 of [4], then

$$
\lim _{n \rightarrow \infty} P\left\{S_{n} / \hat{\sigma}_{n} \leqq x\right\}=\Phi(x) .
$$

The following theorem is a generalization of Theorem 6 .

THEOREM 8. Let $\left\{X_{k}\right\}$ be an absolutely fair sequence of random variables satisfying (1.9) and the conditions of Theorem 7. Assume also that the following conditions are satisfied: $\hat{\sigma}_{n} \rightarrow \infty$ as $n \rightarrow \infty$ such that

$$
\lim _{c \rightarrow 0} \limsup _{n \rightarrow \infty} \frac{\hat{\sigma}_{[n(1+c)]}}{\hat{\sigma}_{n}}=1,
$$

and

$$
\nu_{n} / n \text { converges in probability to } 1 \text { as } n \rightarrow \infty \text {. }
$$

Then

$$
\lim _{n \rightarrow \infty} P\left\{S_{v_{n}} / \hat{\sigma}_{v_{n}} \leqq x\right\}=\Phi(x) .
$$

Proof of Theorem 8. Our sequence $\left\{X_{k}\right\}$ satisfies the central limit theorem with norming factors $\left\{\hat{\sigma}_{n}\right\}$ (Theorem 7). According to Theorem 5 we only have to show that the condition (3.2) is satisfied. A look at the last line of (3.6) and condition (3.8) shows that it is so.

Again, the assumption (3.9) can be replaced by one which says $v_{n} \mid f(n)$ converges in probability to 1 , where $f(n)$ is an arbitrary real valued function which increases monotonically to $\infty$ as $n \rightarrow \infty$.

Now it is of interest to know whether Theorems 6 and 8 are true when $\nu_{n} / n$ converges in probability to a positive random variable $\lambda$ having a discrete (or perhaps not necessarily discrete) distribution, instead of converging to a positive constant. The answer to this question is in the affirmative for the sequence of partial sums $\left\{S_{n}\right\}$ of absolutely fair sequences satisfying (1.9) is a mixing sequence with the limiting unit normal distribution $\Phi(x)$, as will be seen in Theorems 9 and 13.

In general terminology, Rényi [9] calls a sequence of random variables $\left\{\eta_{n}\right\}$ a 
mixing sequence with the limiting distribution function $F(x)$ if for every event $B \in \mathscr{B}$ with $P(B)>0$ and for every real $x$ which is a point of continuity of $F(x)$ we have

$$
\lim _{n \rightarrow \infty} P\left\{\eta_{n} \leqq x \mid B\right\}=F(x),
$$

where, in general, $P(A \mid B)$ is the conditional probability of $A$ given $B(A \in \mathscr{B}$, $B \in \mathscr{B})$ and is defined by $P(A B) / P(B), P(B)>0$.

Let $\left\{X_{k}\right\}$ be a sequence of random variables, define $\left\{S_{n}\right\}$ by (1.2), and let this sequence satisfy the central limit theorem with norming factors $\left\{b_{n}\right\}$, that is to say we have (3.1). There exists then a positive integer $n_{0}$ such that $P\left\{S_{n} / b_{n} \leqq x\right\}>0$ for $n \geqq n_{0}$. It follows then from Theorems 1 and 2 of Rényi's paper [9] that in order to prove that $\left\{S_{n} / b_{n}\right\}$ is a mixing sequence with the limiting unit normal distribution $\Phi(x)$, it is sufficient to show

$$
\lim _{n \rightarrow \infty} P\left\{S_{n} / b_{n} \leqq x \mid S_{k} / b_{k} \leqq x\right\}=\Phi(x)
$$

for any $k \geqq n_{0}$.

THEOREM 9. Let $\left\{X_{j}\right\}$ be an absolutely fair sequence of random variables satisfying (1.9) with $\sigma_{j}^{2}=\sigma_{1}^{2}>0, j \geqq 2$. Then for any $k \geqq n_{0}$, where $n_{0}$ is such that

$$
P\left\{S_{n} / n^{1 / 2} \sigma_{1} \leqq x\right\}>0 \quad \text { for } n \geqq n_{0},
$$

we have

$$
\lim _{n \rightarrow \infty} P\left\{S_{n} / n^{1 / 2} \sigma_{1} \leqq x \mid S_{k} / k^{1 / 2} \sigma_{1} \leqq x\right\}=\Phi(x),
$$

that is the sequence $\left\{S_{n} / n^{1 / 2} \sigma_{1}\right\}$ is mixing with the unit normal distribution $\Phi(x)$.

Proof of Theorem 9. We have already remarked that our sequence of random variables satisfies the central limit theorem with norming factors $\left\{n^{1 / 2} \sigma_{1}\right\}$, that is we have (3.5). Therefore there exists a positive integer $n_{0}$ as postulated in the statement of this theorem. In order to verify (3.13) we need the following lemma (see e.g. [3, p. 254]).

LEMMA 1. If $\delta_{n}$ and $\varepsilon_{n}$ are random variables such that $\lim _{n \rightarrow \infty} P\left\{\delta_{n} \leqq x\right\}=F(x)$ at every point of continuity $x$ of the distribution function $F(x)$, and $\varepsilon_{n}$ converges in probability to zero, then $\lim _{n \rightarrow \infty} P\left\{\delta_{n}+\varepsilon_{n} \leqq x\right\}=F(x)$.

It follows from Lemma 1 and (3.5) that

$$
\lim _{n \rightarrow \infty} P\left\{\left(S_{n}-S_{k}\right) / n^{1 / 2} \sigma_{1} \leqq x\right\}=\Phi(x) .
$$

Now we would like to show

$$
\lim _{n \rightarrow \infty}\left\{P\left(S_{n}-S_{k}\right) / n^{1 / 2} \sigma_{1} \leqq x \mid S_{k} / k^{1 / 2} \sigma_{1} \leqq x\right\}=\Phi(x) .
$$


Define the probability space $\left(\Omega, \mathscr{B}, P^{*}\right)$ with $P^{*}(B)=P\left(B \mid S_{k}^{*} \leqq x\right), B \in \mathscr{B}$ and $S_{k}^{*}=S_{k} / k^{1 / 2} \sigma_{1}$, and consider the characteristic function

$$
\begin{aligned}
E\left\{\exp \left(i t\left(S_{n}-S_{k}\right) / n^{1 / 2} \sigma_{1}\right) \mid S_{k}^{*}\right\} & \\
= & E\left(E\left\{\exp \left(i t\left(S_{n}-S_{k}\right) / n^{1 / 2} \sigma_{1} \mid X_{1}, \ldots, X_{n-1}\right\} \mid S_{k}^{*}\right) .\right.
\end{aligned}
$$

The equality of these two forms follows from the fact that the $\sigma$-field of $\omega$-sets determined by conditions on $X_{1}, \ldots, X_{n-1}$ contains the $\sigma$-field of $\omega$-sets determined by conditions on $S_{k}^{*}, k<n$.

Now the right-hand side of (3.16) is equal to

$$
\begin{aligned}
E\left(\exp \left(i t \frac{X_{k+1}+\cdots+X_{n-1}}{n^{1 / 2} \sigma_{1}}\right)\right. & \left.E\left\{\exp \left(i t X_{n} / n^{1 / 2} \sigma_{1}\right) \mid X_{1}, \ldots, X_{n-1}\right\} \mid S_{k}^{*}\right) \\
= & E\left(\exp \left(i t \frac{X_{k+1}+\cdots+X_{n-1}}{n^{1 / 2} \sigma_{1}}\right)\left\{1-\frac{t^{2}}{2 n}+o\left(\frac{t^{2}}{n}\right)\right\} \mid S_{k}^{*}\right) \\
& =\left\{1-\frac{t^{2}}{2 n}+o\left(\frac{t^{2}}{n}\right)\right\} E\left(\exp \left(i t \frac{X_{k+1}+\cdots+X_{n-1}}{n^{1 / 2} \sigma_{1}}\right) \mid S_{k}^{*}\right) \\
& \vdots \\
& =\left\{1-t^{2} / 2 n+o\left(t^{2} / n\right)\right\}^{n-k} \rightarrow \exp \left(-t^{2} / 2\right) \quad \text { as } n \rightarrow \infty .
\end{aligned}
$$

In line two of above argument we used the condition (1.9) with $\sigma_{j}^{2}=\sigma_{1}^{2}>0$, $j \geqq 2$, in $E\left(\exp \left(\right.\right.$ it $\left.\left.X_{n}\right) \mid X_{1}, \ldots, X_{n-1}\right)=1-\frac{1}{2} \sigma_{1}^{2} t^{2}+o\left(t^{2}\right)$ and then we applied, mutatis mutandis, the equality (3.16) repeatedly. Letting $n \rightarrow \infty$ we see that (3.15) is true.

Applying again Lemma 1 with $\delta_{n}=\left(S_{n}-S_{k}\right) / n^{1 / 2} \sigma_{1}$ and $\varepsilon_{n}=S_{k} / n^{1 / 2} \sigma_{1}$ on the probability space $\left(\Omega, \mathscr{B}, P^{*}\right)$, (3.13) follows through (3.15) and Theorem 9 is proved.

Using Theorem 9 we can generalize Theorem 6 as follows.

THEOREM 10. Let $\left\{X_{k}\right\}$ be an absolutely fair sequence of random variables satisfying the condition (1.9) with $\sigma_{k}^{2}=\sigma_{1}^{2}>0, k \geqq 2$. If $\nu_{n} / n$ (or $\nu_{n} \mid f(n)$, where $f(n)$ is an arbitrary real valued function which increases monotonically to $\infty$ as $n \rightarrow \infty$ ) converges in probability to a positive random variable, having a discrete distribution, as $n \rightarrow \infty$, then

$$
\lim _{n \rightarrow \infty} P\left\{S_{v_{n}} / \nu_{n}^{1 / 2} \sigma_{1} \leqq x\right\}=\Phi(x) .
$$

Proof of Theorem 10. Theorem 9 tells us that the sequence $\left\{S_{n} / n^{1 / 2} \sigma_{1}\right\}$ is mixing with the unit normal distribution. Mixing sequences of random variables have the remarkable property that in the limit they are independent of any random variable (Rényi, statement (2.8) of [9]). Thus, as a consequence of Theorem 9, we have

CoROllary 1. Retain the assumptions of Theorem 9 and let $\delta$ be any random variable. If $P\{\delta \leqq y\}>0$, then

$$
\lim _{n \rightarrow \infty} P\left\{S_{n} / n^{1 / 2} \sigma_{1} \leqq x \mid \delta \leqq y\right\}=\Phi(x)
$$


For the sequence of random variables of Theorem 10 we already verified the Anscombe condition (3.2) in the argument (3.6), using Kolmogorov's inequality for martingales. These two facts (Corollary 1 and the verified condition (3.2)) together with Rényi's proof of his Theorem 2 in [10] imply Theorem 10 as follows. Replace the assumption that the sequences of random variables in question are mutually independent by the one that they are absolutely fair and satisfy (1.9) with $\sigma_{k}^{2}=\sigma_{1}^{2}>0, k \geqq 2$. Replace Lemma 4 of Rényi's proof of his Theorem 2 in [10] by Corollary 1 above. This carries us till statement (13) of his proof, and a statement (13) type version in our situation follows from the already verified condition (3.2) for our partial sums $\left\{S_{n}\right\}$. The rest of Rényi's proof of his Theorem 2 of [10] remains valid, mutatis mutandis, for Theorem 10 of this paper and this completes its proof too.

The lines of proof of Theorem 10 suggest the following general statement.

THEOREM 11. Let $\left\{\eta_{n}\right\}$ be a mixing sequence of random variables with the limiting distribution function $F(x)$, that is relation (3.11) holds. Let $v_{n} \mid f(n)$, where $f(n)$ is as before, converge in probability to a positive discrete random variable $\lambda$ as $n \rightarrow \infty$. Let the sequence $\left\{\eta_{n}\right\}$ satisfy the Anscombe condition (3.2). Then

$$
\lim _{n \rightarrow \infty} P\left\{\eta_{v_{n}} \leqq x\right\}=F(x),
$$

for every real $x$ which is a point of continuity of $F(x)$.

The proof is again analogous to the one given by Rényi in [10] for Theorem 2 . Theorem 10 can be generalized as follows.

THEOREM 12. Retain the assumptions of Theorem 10 about the sequence $\left\{X_{k}\right\}$. Then the statement (3.17) holds if $\nu_{n} / n$ (or $v_{n} / f(n)$ with $f(n)$ as before) converges in probability to a positive, not necessarily discrete random variable $\lambda$ as $n \rightarrow \infty$.

The proof of this theorem will be given at the end of this section.

Next we generalize Theorem 8 the way we generalized Theorem 6 in Theorem 10 . In order to do so we need the following generalization of Theorem 9.

THEOREM 13. Let $\left\{X_{n}\right\}$ be an absolutely fair sequence of random variables satisfying (1.9) and the conditions of Theorem 7. Define $\hat{\sigma}_{n}^{2}$ as in (1.10) and assume also that $\hat{\sigma}_{n} \rightarrow \infty$ as $n \rightarrow \infty$. Then for any $k \geqq n_{0}$, where $n_{0}$ is such that $P\left\{S_{n} / \hat{\sigma}_{n} \leqq x\right\}>0$ for $n \geqq n_{0}$, we have

$$
\lim _{n \rightarrow \infty} P\left\{S_{n} / \hat{\sigma}_{n} \leqq x \mid S_{k} / \hat{\sigma}_{k} \leqq x\right\}=\Phi(x)
$$

that is the sequence $\left\{S_{n} / \hat{\sigma}_{n}\right\}$ is mixing with the unit normal distribution function $\Phi(x)$.

Proof of Theorem 13. Our sequence of random variables satisfies the central limit theorem with norming factors $\left\{\hat{\sigma}_{n}\right\}$ (Theorem 7). There exists, therefore, a 
positive integer $n_{0}$ as postulated in the statement of this theorem. From the proof of Theorem 8 it follows that in order to verify (3.20) it is sufficient to verify

$$
\lim _{n \rightarrow \infty} P\left\{\left(S_{n}-S_{k}\right) / \hat{\sigma}_{n} \leqq x \mid S_{k} / \hat{\sigma}_{k} \leqq x\right\}=\Phi(x),
$$

for any $k \geqq n_{0}$.

Using the condition (1.9) and the method of proof of Theorem 9 we get, on neglecting error terms,

$$
\begin{aligned}
\phi_{j}(t) & =E\left\{\exp \left(i t\left(S_{j}-S_{k}\right)\right) \mid S_{k}\right\} \\
& =\left(1-t^{2} \sigma_{j}^{2} / 2\right) E\left\{\exp \left(i t\left(X_{k+1}+\cdots+X_{j-1}\right)\right) \mid S_{k}\right\}, \quad j>k .
\end{aligned}
$$

So, approximately, we have

$$
\log \phi_{j}(t)-\log \phi_{j-1}(t)=-t^{2} \sigma_{j}^{2} / 2, \quad k<j \leqq n .
$$

Adding these equations, we get

$$
\log \phi_{n}(t)=\log E\left\{\exp \left(i t\left(S_{n}-S_{k}\right)\right) \mid S_{k}\right\}=-\frac{t^{2}}{2} \sum_{j=k+1}^{n} \sigma_{j}^{2}=-\frac{t^{2}}{2}\left(\hat{\sigma}_{n}^{2}-\hat{\sigma}_{k}^{2}\right) .
$$

Therefore, approximately, we have

$$
\log \phi_{n}\left(t / \hat{\sigma}_{n}\right)=-\left(t^{2} / 2\right)\left(1-\hat{\sigma}_{k}^{2} / \hat{\sigma}_{n}^{2}\right)
$$

Now given that the sequence $\left\{X_{k}\right\}$ of Theorem 13 satisfies the central limit theorem with norming factors $\left\{\hat{\sigma}_{n}\right\}$ (Theorem 7), the neglected error terms in (3.22) are uniformly small in $t$ and in the $\left\{X_{k} / \hat{\sigma}_{n}\right\}$ distributions involved as $n \rightarrow \infty$, and $\log \phi_{n}\left(t / \hat{\sigma}_{n}\right) \rightarrow-t^{2} / 2$ as $n$ and $\hat{\sigma}_{n} \rightarrow \infty$, for any $k \geqq n_{0}$. This verifies (3.21) and Theorem 13 is proved.

Having this result the following generalization of Theorem 8 is immediately available.

THEOREM 14. Retain the assumptions of Theorem 8 about the sequence $\left\{X_{k}\right\}$ and its condition (3.8). If $\nu_{n} / n$ (or $\nu_{n} / f(n)$, where $f(n)$ is as before) converges in probability to a positive random variable $\lambda$, having a discrete distribution, as $n \rightarrow \infty$, then (3.10) holds.

This theorem can be proved directly exactly the same way as Theorem 10, or we can look at it as a special case of Theorem 11 (in proving Theorem 8 we observed that the Anscombe condition (3.2) is satisfied in this case and Theorem 13 ensures mixing of the sequence $\left\{X_{k}\right\}$ with the distribution function $\Phi(x)$ ).

A not entirely trivial generalization of Theorem 14 is as follows.

THEOREM 15. Retain the assumption of Theorem 8 about the sequence $\left\{X_{k}\right\}$. Let the sequence $\left\{\hat{\sigma}_{n}\right\}$ be slowly oscillating in the sense of Karamata; that is to say,

$$
\hat{\sigma}_{n}=n^{\alpha} L(n), \quad \alpha>0,
$$

where for any $c>0, L([c n]) / L(n) \rightarrow 1$ as $n \rightarrow \infty$. If $\nu_{n} / n\left(\right.$ or $\nu_{n} / f(n)$ with $f(n)$ as before $)$ 
converges in probability to a positive, not necessarily discrete random variable $\lambda$ as $n \rightarrow \infty$, then (3.10) holds.

The proof of Theorem 15 is similar to that of Theorem 12 whose proof will be given after the following preliminary results (Theorems 16 and 17).

THEOREM 16. Let $\left\{X_{k}\right\}$ be an absolutely fair sequence of random variables satisfying (1.9) and the conditions of Theorem 7. Let $\hat{\sigma}_{n} \rightarrow \infty$ as $n \rightarrow \infty$. Then given any event $A \in \mathscr{B}$ with $P(A)>0$, we have

$$
\lim _{n \rightarrow \infty} E\left\{S_{n}^{2}\left|\hat{\sigma}_{n}^{2}\right| A\right\}=1, \quad \text { as } n \rightarrow \infty .
$$

In case the random variables $\left\{X_{k}\right\}$ are mutually independent, Theorem 16 is a special case of Theorem 6 of J. Mogyoródi in [8] and its proof differs only notationally from that of his Theorem 6. As a matter of fact we have here $E\left\{S_{n}^{2} / \hat{\sigma}_{n}^{2}\right\}$ $=\int_{-\infty}^{+\infty} x^{2} d \Phi(x)=1$ and $F_{n}(x)$ converges to $\Phi(x)$ as $n \rightarrow \infty$, where $F_{n}(x)$ is the distribution function of the random variable $S_{n} / \hat{\sigma}_{n}$ (Theorem 7). These two facts carry us through, mutatis mutandis, when proving our Theorem, till after the first statement after statement (15) in Mogyoródi's proof of his above mentioned theorem. According to Theorem 13 of this paper we also have $F_{n}(x \mid A) \rightarrow \Phi(x)$, and Mogyoródi's last lines of proof remain valid, mutatis mutandis, in our case too.

Theorem 16 is a generalization of J. Mogyoródi's Theorem 6 in [8] in the sense that we talk about absolutely fair random variables instead of mutually independent ones but, it is also more restricted than his theorem for we restrict ourselves to the unit normal distribution function $\Phi(x)$ as our limiting distribution function of $F_{n}(x)$. This is because we proved the mixing property for the sequence $\left\{S_{n} / \hat{\sigma}_{n}\right\}$ with the unit normal distribution function only (Theorem 13). The question of mixing of a martingale sequence of random variables $\left\{S_{n}\right\}$ with any possible limiting distribution function $F(x)$ is still open. Should it be true however that partial sums of absolutely fair random variables were mixing with any possible limiting distribution function $F(x)$ in the sense of Rényi's definition (3.11), then Mogyoródi's Theorem 6 in [8] would also remain true for them.

THEOREM 17. Let $\left\{\xi_{k}\right\}$ be any sequence of random variables with $E\left(\xi_{1}\right)=\mu_{1}$, and write $E\left(\xi_{k} \mid \xi_{1}, \ldots, \xi_{k-1}\right)=\mu_{k}$, for $k=1,2, \ldots, \xi_{0}=0$. Put $X_{k}=\xi_{k}-\mu_{k}$ (now $\left\{X_{k}\right\}$ is an absolutely fair sequence) and let these $X_{k}$ 's satisfy the condition (1.9) and also the conditions of Theorem 7. Let $B$ be any event having positive probability. Then there exists an integer $n_{0}=n_{0}(B)$ such that for $n \geqq n_{0}$,

$$
P\left\{\max _{1 \leqq k \leqq n}\left|\sum_{i=1}^{k} X_{i}\right| \geqq \varepsilon \hat{\sigma}_{n}, B\right\} \leqq \frac{3}{\varepsilon^{2}}\{P(B)\}^{1 / 2},
$$

where $\varepsilon$ is arbitrary positive number. 
Theorem 17 is a Kolmogorov type inequality for martingales. In case the random variables $\left\{\xi_{k}\right\}$ are mutually independent, it is a special case of Theorem 7 of Mogyoródi in [8]. The content of the paragraph preceding Theorem 17 concerning the relation between Theorem 16 and Mogyoródi's Theorem 6 of [8] remains valid regarding the relation between Theorem 17 and Mogyoródi's Theorem 7 of [8] too.

Proof of Theorem 17. The proof of Theorem 17 is similar to that of Theorem 7 of Mogyoródi in [8]. We are going to introduce some notation here and will only give those lines of proof afterwards which differ from his appropriate ones.

Let $S_{k}=\sum_{1}^{k} X_{i}$. The summands of $S_{k}$ form an absolutely fair sequence and the partial sums $\left\{S_{k}\right\}$ form a martingale sequence. Let $\gamma$ be the indicator of the event $B$. Let $A_{k}, k=2, \ldots, n$, be the event

$$
\left|S_{j}\right|<\varepsilon \hat{\sigma}_{n}, \quad j=1,2, \ldots, k-1 \quad \text { and } \quad\left|S_{k}\right| \geqq \varepsilon \hat{\sigma}_{n}
$$

and let $A_{1}$ be the event $\left|S_{1}\right| \geqq \varepsilon \hat{\sigma}_{n}$. Further, let $\alpha_{k}$ be the indicator of the event $A_{k}$. The lines of the first paragraph of Mogyoródi's proof remain true when using the language and notation of Theorem 17. Now we write the random variable $\beta_{j}$ of Mogyoródi's proof in terms of our notation as

$$
\beta_{j}=X_{j} \sum_{k=1}^{j-1} \alpha_{k} S_{k}, \quad j=2,3, \ldots, n
$$

and are going to show that $E\left(\beta_{j} \beta_{i}\right)=0$ for $j<i$.

Consider

$$
E\left(\beta_{j} \beta_{i}\right)=E\left\{\left(X_{j} \sum_{k=1}^{j-1} \alpha_{k} S_{k}\right)\left(X_{i} \sum_{k=1}^{i-1} \alpha_{k} S_{k}\right)\right\}, \quad j<i
$$

The right-hand side of (3.23) can be written as

$$
\begin{aligned}
E\left[E\left\{\left(X_{j} \sum_{k=1}^{j-1} \alpha_{k} S_{k}\right)\left(X_{i} \sum_{k=1}^{i-1} \alpha_{k} S_{k}\right) \mid X_{1}, \ldots, X_{i-1}\right\}\right] \\
=E\left[\left(X_{j} \sum_{k=1}^{j-1} \alpha_{k} S_{k}\right)\left(\sum_{k=1}^{i-1} \alpha_{k} S_{k}\right) E\left\{X_{i} \mid X_{1}, \ldots, X_{i-1}\right\}\right] \\
=0, \quad \text { for the sequence }\left\{X_{i}\right\} \text { is absolutely fair. }
\end{aligned}
$$

Thus, when the $\beta_{j}$ 's are defined in terms of absolutely fair random variables, the above argument replaces paragraph 2 of Mogyoródi's proof. 
Next we consider

$$
\begin{aligned}
E\left(\beta_{j}^{2}\right) & =E\left\{X_{j}^{2}\left(\sum_{k=1}^{j-1} \alpha_{k} S_{k}\right)^{2}\right\} \\
& =E\left[E\left\{X_{j}^{2}\left(\sum_{k=1}^{j-1} \alpha_{k} S_{k}\right)^{2} \mid X_{1}, \ldots, X_{j-1}\right\}\right] \\
& =E\left[\left(\sum_{k=1}^{j-1} \alpha_{k} S_{k}\right)^{2} E\left\{X_{j}^{2} \mid X_{1}, \ldots, X_{j-1}\right\}\right] \\
& =\sigma_{j}^{2} E\left[\left(\sum_{k=1}^{j-1} \alpha_{k} S_{k}\right)^{2}\right] \\
& =\sigma_{j}^{2} \sum_{k=1}^{j-1} E\left(\alpha_{k}^{2} S_{k}^{2}\right) .
\end{aligned}
$$

The fourth line of (3.24) follows from the property of $X_{j}$ 's that they satisfy the condition (1.9), and its last line is the result of the fact that $\alpha_{i} \alpha_{j}=0$ if $i \neq j$. Also, because of the submartingale property of the sequence $\left\{S_{k}^{2}\right\}$, we have

$$
E\left\{S_{n}^{2} \mid S_{1}^{2}, \ldots, S_{k}^{2}\right\} \geqq S_{k}^{2}, \quad k<n .
$$

Therefore,

$$
\begin{aligned}
E\left(\alpha_{k}^{2} S_{k}^{2}\right) & \leqq E\left\{\alpha_{k}^{2} E\left(S_{n}^{2} \mid S_{1}^{2}, \ldots, S_{k}^{2}\right)\right\} \\
& =E\left\{E\left(S_{n}^{2} \mid S_{1}^{2}, \ldots, S_{k}^{2}\right) \mid A_{k}\right\} P\left(A_{k}\right) \\
& =E\left(S_{n}^{2} \mid A_{k}\right) P\left(A_{k}\right) .
\end{aligned}
$$

Putting (3.24) and (3.25) together we get

$$
E\left(\beta_{j}^{2}\right) \leqq \sigma_{j}^{2} \sum_{k=1}^{j-1} E\left(S_{n}^{2} \mid A_{k}\right) P\left(A_{k}\right) \leqq \sigma_{j}^{2} E\left(S_{n}^{2}\right)=\sigma_{j}^{2} \hat{\sigma}_{n}^{2} .
$$

The above argument leading up to (3.26) and the statement (3.26) itself takes the place of paragraph 3 in Mogyoródi's proof of his Theorem 7 in [8] in our case. The lines of his proof after paragraph 3, leading up to and including his statement (17) remain valid for Theorem 17 of this paper too, and the last few lines of his proof hold true in the sense of our Theorem 16. This completes the proof of Theorem 17.

Proof of Theorem 12. Theorems 13, 16 and 17 together with Mogyoródi's proof of his Theorem 3 in [6] imply Theorem 12 as follows. Replace Theorem 4 (a theorem of Rényi [9]) in Mogyoródi's paper [8] by Theorem 13 (or by Theorem 9) of our paper. Also replace Theorems 6 and 7 of Mogyoródi's paper [8] by Theorems 16 and 17 of our paper, respectively, and repeat the argument of his proof of Theorem 3 in [8], mutatis mutandis, for an absolutely fair sequence of random variables which also satisfy (1.9), quoting when necessary Theorem 13 or 16 or 17 , as the case may be, instead of the replaced ones. 
ACKNOWLEDGEMENT. The author would like to thank the referee for many valuable remarks and Professors D. A. Dawson, R. Fischler and P. Greiner of the Kingston Summer Research Institute, 1966 for many useful discussions.

\section{REFERENCES}

1. F. J. Anscombe, Large-sample theory of sequential estimation, Proc. Cambridge Philos. Soc. 48 (1952), 600-607.

2. J. R. Blum, D. L. Hanson, and J. I. Rosenblatt, On the central limit theorem for the sum of a random number of independent random variables, $\mathrm{Z}$. Wahrscheinlichkeitstheorie und Verw. Gebiete 1 (1963), 389-393.

3. H. Cramér, Mathematical methods of statistics, Princeton Math. Series, Vol. 9, Princeton Univ. Press, Princeton, N. J., 1956.

4. J. L. Doob, Stochastic processes, Wiley, New York, 1953.

5. W. Feller, An introduction to probability theory and its applications, Vol. II, Wiley, New York, 1966.

6. J. Hájek and A. Rényi, Generalization of an inequality of Kolmogorov, Acta Math. Acad. Sci. Hungar. 6 (1955), 281-283.

7. M. Loève, Probability theory, Van Nostrand, New York, 1960.

8. J. Mogyoródi, $A$ central limit theorem for the sum of a random number of independent random variables, Publ. Math. Inst. Hungar. Acad. Sci. 7A (1963), 409-424.

9. A. Rényi, On mixing sequences of sets, Acta Math. Acad. Sci. Hungar. 9 (1958), 215-228.

10. - On the central limit theorem for the sum of a random number of independent random variables, Acta Math. Acad. Sci. Hungar. 11 (1960), 97-102.

MCGILL UNIVERSITY, Montreal, Canada 